\title{
Multimedia CONTENT AND LABORATORY ENHANCEMENTS FOR Courses in Digital Systems AND CoMputer ArChitecture
}

\author{
Naraig Manjikian ${ }^{1}$ and Valerie Sugarman ${ }^{2}$ \\ ${ }^{1}$ Department of Electrical and Computer Engineering, Queen's University, Kingston, Ontario K7L 3N6 \\ ${ }^{2}$ David R. Cheriton School of Computer Science, University of Waterloo, Waterloo, Ontario N2L 3G1 \\ naraig.manjikian@queensu.ca
}

\begin{abstract}
This paper describes multimedia content and laboratory enhancements for courses in digital systems and computer architecture at Queen's University. The laboratory enhancements for the computer architecture course reflect new equipment with field-programmable logic chip technology for continuity from the preceding digital systems course. New laboratory exercises on processor implementation rely on the multimedia content and reinforce the technical link to the preceding logic course. Student usage of multimedia content in the two courses was monitored with a learning management system, revealing averages of almost 10 accesses per student in the digital systems course and over 15 accesses per student in the computer architecture course. Some students made no accesses, but others made 20 to 30 accesses. Finally, results from surveys conducted in the courses to obtain feedback from students indicate that the majority of respondents obtained a learning benefit from the content.
\end{abstract}

Keywords: Multimedia, laboratory design, digital systems, computer architecture, field-programmable logic.

\section{INTRODUCTION}

This paper describes learning enhancements for core second-year engineering courses in digital systems and computer architecture at Queen's University. A primary motivation has been a decision to revamp the content and laboratory equipment for the computer architecture course for cohesion and continuity across multiple courses. Multimedia content with high production values has been generated to cover important concepts and practical considerations in both courses as a learning aid for students. Furthermore, new laboratory exercises have been created for the computer architecture course, and these exercises are complemented by relevant multimedia content.

In other work, Toral et al. [5] have described the provision of Web-based multimedia content as a learning resource for students, and they also tracked usage of that content by students. Butz et al. [2] and Poli et al. [4] de- scribe the use of on-line tutoring with multimedia content and different degrees of interaction and sophistication for those interactions. In this paper, the selected approach is one where students are self-guided in their usage of available multimedia content to pursue review and reinforcement of concepts. Tracking of usage is also considered.

The problem that the project discussed in this paper seeks to address is to enhance student learning of fundamental concepts for digital systems and computer architecture, in combination with practical application of those concepts using contemporary hardware technology and software tools. This problem is underscored by the limited background of many students in the specific theory, technology, and tools that are relevant for introductory engineering courses in digital systems and computer architecture. In contrast, students may possess somewhat more background in mathematics and physics for introductory university-level engineering courses that are closer to these domains of knowledge.

Candidate solutions for addressing the problem include studio teaching and increased contact time in tutorial and laboratory sessions, as well as the provision of additional independent learning resources such as multimedia content. Increased contact time and studio teaching are subject to practical constraints related to curriculum, timetabling, and physical space. For this project, the methodologies chosen include the creation of multimedia content and enhanced laboratory exercises with a connection to the multimedia content. The rationale for this approach is that it is not subject to the aforementioned constraints; the existing curriculum, timetable, and space are complemented with additional material.

The results of the project in this paper include multimedia presentations for two courses, along with templates and other resources such as custom animation software to support generation of future multimedia content. New laboratory exercises that link concepts across courses have also been created. The results also include tracking of student usage of multimedia content and generally positive feedback from students. The remainder of this paper discusses these aspects in more detail. 


\section{CONTEXT FOR COURSES}

The Department of Electrical and Computer Engineering at Queen's University is responsible for the following sequence of related undergraduate courses:

- ELEC 271 Digital Systems

- ELEC 274 Computer Architecture

- ELEC 371 Microprocessor Interfacing and Embedded Systems

- ELEC 374 Digital Systems Engineering

- ELEC 470 Computer System Architecture

ELEC 271, 274, and 371 are core requirements for all students in the Electrical Engineering program and Computer Engineering program. ELEC 374 is a core course for Computer Engineering, and ELEC 470 is an elective course for Electrical Engineering and for Computer Engineering. ELEC 271, 274, and 371 are also included in certain program options in Math and Engineering, Engineering Physics, and Mechanical Engineering. Thus, students in five of the ten undergraduate programs in the Faculty of Engineering and Applied Science at Queen's University are enrolled every year in the two courses that are the focus of this paper, ELEC 271, and ELEC 274.

ELEC 271 is a lecture-only course on digital logic, with an emphasis on implementation in fieldprogrammable gate-array (FPGA) chips. This emphasis is reflected in the textbook for the course [1]. Logic design software and FPGA-based equipment are described and demonstrated in lectures, but practical activities are undertaken in a separate laboratory course that also serves an introductory course on electric circuits. For almost two decades, FPGA technology from Altera Corp. has been used in second-year logic laboratory activities at Queen's University. Equipment in current use is shown in Fig. 1. The full-featured Altera Quartus II software for logic design is available at no cost for students to install on their own computers for learning outside of the laboratory.

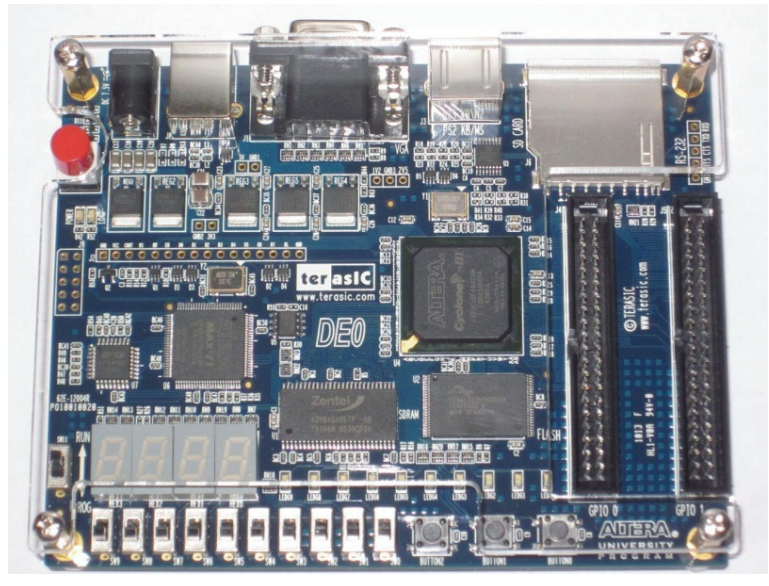

Fig. 1. Altera DE0 board with Cyclone III FPGA chip.
On the other hand, ELEC 274 on computer architecture has a dedicated laboratory. The subsequent course ELEC 371 on microprocessor/embedded systems also has a dedicated laboratory. ELEC 274 and ELEC 371 have been revised together to use the 32-bit Altera Nios II processor architecture [6] implemented in the FPGA chip on the board shown in Fig. 1. In this manner, there is continuity in the application of contemporary FPGA technology after the digital systems course. The revision of ELEC 274 and ELEC 371 also reflects the adoption of a new textbook [3] that is used in both courses. These courses employ the Altera Quartus II software for logic design, and also the Altera adaptation of the open-source GNU software to generate code for the 32-bit processor. Again, this software is available to students at no cost.

Therefore, the overall context for the enhancements to ELEC 271 and ELEC 274 discussed in this paper is an environment for students to learn fundamental concepts while also acquiring a strong practical appreciation of relevant technology and software tools for digital logic and computer architecture. Multimedia content can aid in learning of course concepts and in providing tutorial guidance for usage of software and equipment. Appropriate laboratory exercises can be complemented effectively by multimedia content for overall learning enhancement.

\section{FACILITIES, EQUIPMENT, AND TOOLS FOR MULTIMEDIA PRODUCTION}

This section provides a brief overview of the facilities, equipment, and software used in the production of the multimedia content described in this paper.

Recording Studio: Queen's University operates a Podcasting Studio with audio/video equipment that is freely available for academic use. This facility was used for audio narration for several weeks until nearby heavy construction required switching to use of personal equipment.

Personal Audio/Video Equipment: A personal highdefinition video camera was used to obtain video on preparation and use of laboratory hardware. When construction prevented subsequent use of the Podcasting Studio, this camera and a high-quality external microphone were used in a quiet conference room for subsequent narration. Audio was separated from the unnecessary video with the free Pazera software. Quality with the external microphone was close to that from the Podcasting Studio.

Graphics Drawing Software: The open-source xfig program was selected to generate diagrams and figures. Templates with a $16 \times 9$ aspect ratio were created for highdefinition multimedia content. With $x$ fig allowing objects to be assigned to different layers, these templates included a background watermark and the Queen's University crest as optional items to include in output images.

Dynamic Screen Capture Software: To capture user interaction with software tools and dynamic content on a

CEEA13; Paper 054

Montreal, QC; June 17-20, 2013 
computer screen into digital video files for subsequent editing and multimedia integration, the CaptureWiz software from PixelMetrics was used for this project.

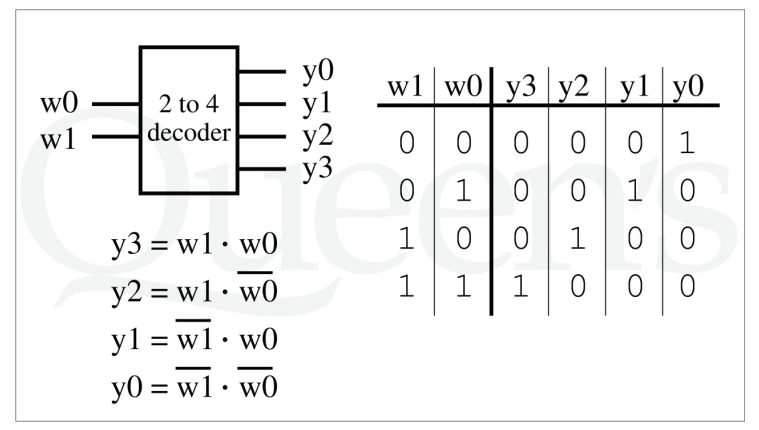

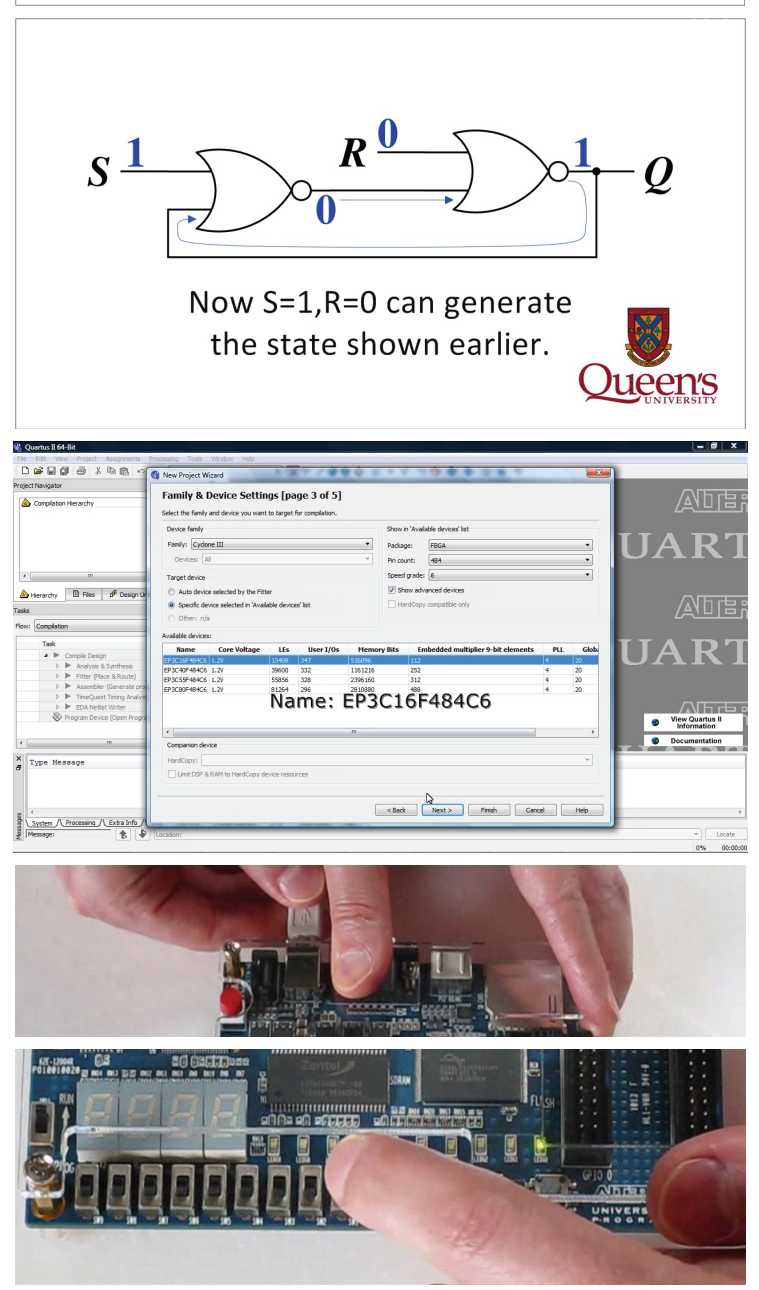

Fig. 2. Sample multimedia content for digital systems

Custom Animation Software: This project relied on a direct approach to support simple animations consisting of lines, arrows, boxes, and text. A custom library of graphical animation subroutines was prepared for opensource X Windows in the cygwin environment. Animation sequences were defined by calling these subroutines with appropriate delays, and their executions were then captured into video files using the CaptureWiz tool.

Image Montage Editing Software: This project made use of the Microsoft Photo Story 3 software tool for producing video files of dynamic montages from static images in order to enhance the production values of the content. The zoom-in/zoom-out capability of this tool was the primary feature that was utilized. Other video editing software may also provide such a capability, but Photo Story 3 provided a desirable precision for zoom control.

Full Multimedia Editing Software: This project relied on Windows Movie Maker 6 from Microsoft for editing and integration of audio/video content. WMM6 offers a timeline view that displays audio levels and permits precise alignment of audio/video segments. In contrast, the more recent Live Movie Maker software from Microsoft does not offer comparable features for precise editing.

\section{MULTIMEDIA CONTENT}

The production of multimedia content for ELEC 271 considered the following concepts and practical considerations related to digital logic and relevant technology:

- Boolean algebra, truth tables, basic logic gates,

- optimization of logic functions,

- $\quad$ standard logic blocks (e.g., encoders/decoders),

- latches, flip-flops, and counters,

- circuit specification for logic design software, simulation, and implementation in FPGA chips.

Twelve multimedia presentations spanning these topics have been produced as part of this project. The presentations range from 3 to 6 minutes in duration, and are therefore focused and concise for efficient independent study outside of the classroom as a complement to conventional full-length lecture exposition. Figure 2 shows screencapture examples from some of this multimedia content. The examples include explanation of logic concepts such as decoders and latches, tutorial guidance on use of software tools for design and simulation, and preparation and usage of equipment for circuit implementation.

The production of multimedia content for ELEC 274 considered the following concepts and practical considerations for computer architecture and programming:

- number representation (e.g., binary, hexadecimal),

- basic assembly-language instructions for performing arithmetic and accessing memory,

- programming loops for computation on array data,

- instructions for supporting subroutine calls,

- programming to support character input/output,

- cycle-by-cycle execution behavior of instructions,

- use of software tools to generate machine code and test its execution on laboratory equipment.

Another twelve multimedia presentations spanning these topics have been produced for this project, and they also 
range from 3 to 6 minutes in typical duration to enable efficient independent study. Figure 3 shows screencapture examples from this content. The examples include cycle-by-cycle explanations of instruction execution, tutorials on software tools for code generation and interfacing with laboratory equipment, and detailed explanation of program behavior tracing register and memory contents.

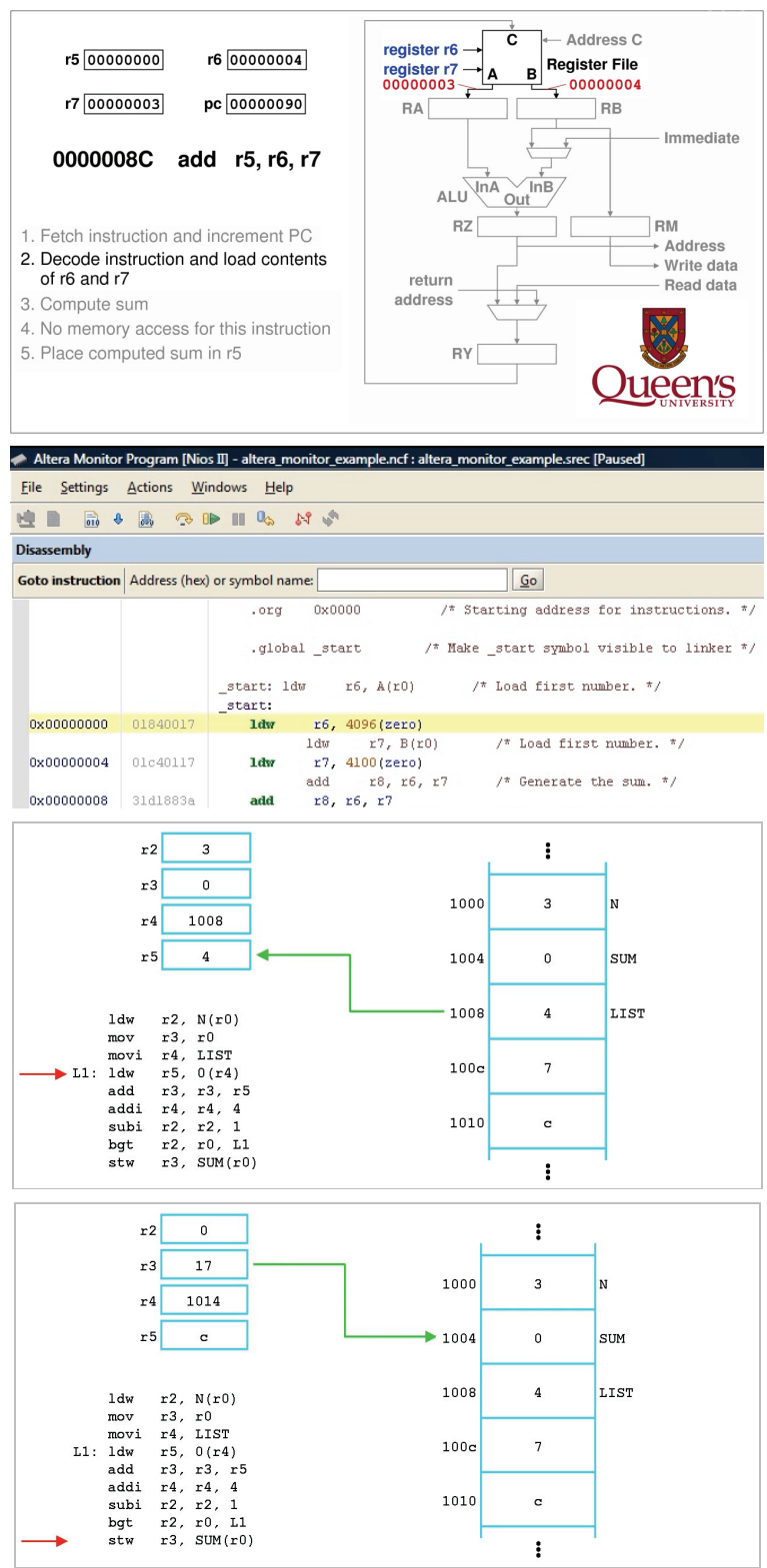

Fig. 3. Sample multimedia for computer architecture

Section 3 described custom animation software for this project. The lower half of Figure 3 shows two screencapture images from an animation sequence produced with this custom software. The lines, arrows, boxes, and text are drawn and erased by calling subroutines in an appropriate sequence with delays. In particular, the arrow on the left that points to the active instruction is moved smoothly up or down to show the progression of instruction execution through straight-line sequencing or branching back to the beginning of the loop. The programmed animation sequence in the lower half of Fig. 3 is recorded in a digital video file using the CaptureWiz tool described in Section 3. That video file is integrated with audio narration to explain the behavior depicted in the animation.

The average time to prepare a multimedia presentation was 12 hours. Content with video of equipment or software tutorials required less time, whereas content with detailed diagrams or animation required more time.

\section{LABORATORY ENHANCEMENTS}

The Altera DE0 board shown in Fig. 1 was adopted for laboratory use in Fall 2011. Prior to that point, laboratory activities for digital logic used the similar, but physically larger, Altera DE2 board. As such, existing logic laboratory exercises have not required major revisions.

The laboratory in ELEC 274, however, previously relied on a graphical software simulator for an 8-bit microcontroller processor architecture that permitted students to observe the simulated execution of machine instructions. No actual hardware was involved. The laboratory in ELEC 371 used a hardware platform based on the same 8-bit processor architecture used in ELEC 274.

A decision to make substantial revisions to ELEC 274 and ELEC 371 has exploited the introduction of the Altera DE0 board as an opportunity to pursue new learning enhancements in a manner that provides continuity across three courses, ELEC 271, 274, and 371. Building on the FPGA-based logic-design experience in ELEC 271 and the use of the Altera DE0 for the related laboratory activity in a separate course, the same hardware platform is now used in the laboratory activity of both ELEC 274 and ELEC 371 with a 32-bit Altera Nios II processor implementation embedded in the FPGA chip.

The focus of this paper is on ELEC 271 and ELEC 274 , and the narrower focus of this section is on ELEC 274. Students in ELEC 274 have six laboratory sessions. With the adoption of the Altera DE0 and its introduction into the laboratory component of ELEC 274, six new laboratory exercises have therefore been developed.

The first exercise is an introduction to assemblylanguage programming and to new software tools (distinct from logic-design tools discussed in ELEC 271) for generating code and testing its execution in the FPGAembedded Nios II processer on the Altera DE0 board.

Three of the remaining five laboratory exercises cover assembly-language programming with nested subroutines, character input/output, and other aspects. These are necessary exercises to progressively increase the exposure of students to these concepts and their practical application. 
The most significant laboratory enhancement for ELEC 274, however, is reflected in two new laboratory exercises on processor implementation. For these exercises, a custom hardware description in the VHDL language has been developed for a simplified 32-bit processor for the Altera Nios II instruction set. This description consists of approximately 400 lines of raw VHDL code, including comments and blank lines for readability. It can be processed by computer-aided design software for logic simulation and implementation in an FPGA chip. Of the 86 instructions defined for the Altera Nios II architecture, the simplified processor only supports 4 instructions: load from memory, store to memory, addition, and unconditional branch. This subset is sufficient for simple loop code to study execution behavior in simulation and also for on-chip implementation in the hardware of Fig. 1.

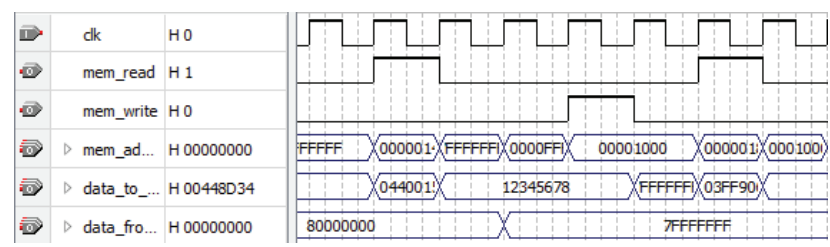

Fig. 4. Waveforms from processor logic simulation

Students are provided with the VHDL description of the processor. Building on logic background from ELEC 271, they study it and relate it to concepts in ELEC 274. In one laboratory exercise, they perform logic synthesis and functional timing simulations to observe the cycleby-cycle behavior for fetching and executing instructions, as shown in Fig. 4. In a second laboratory exercise, they must implement a new instruction by making appropriate modifications and extensions of the VHDL hardware description. In this manner, students effectively integrate knowledge of material from ELEC 271 and ELEC 274.

The laboratory exercises on processor implementation are complemented by the multimedia presentations shown in Fig. 3 for ELEC 274, particularly the top screencapture image for cycle-by-cycle instruction execution. Effective learning by students combines the multimedia content with laboratory activity, along with conventional classroom exposition and independent problem solving.

Finally, the same VHDL description initially explored in ELEC 274 is used again for continuity in the subsequent course ELEC 371 where students introduce new logic for address decoding and memory, as well as parallel ports for input/output capability.

\section{MONITORING ACCESS TO MULTIMEDIA CONTENT}

Queen's University provides the open-source Moodle learning management system for instructors to use in their courses. Multimedia content on a streaming server can be embedded in Moodle course pages. This capability has been used to provide students with access to the multimedia content generated for ELEC 271 and ELEC 274.

For instructors, Moodle can track all student accesses to embedded content. Each time a student views a multimedia presentation within Moodle, the system records the student identifier and date/time information, and an instructor can later generate a detailed report on accesses. Student activity in Moodle was monitored in this way for ELEC 271 in Fall 2011, and ELEC 274 in Winter 2012.

For ELEC 271, Moodle reported nearly 1000 accesses to the multimedia content for the period SeptemberDecember 2011. With an enrolment of 123 students, the average number of accesses was less than 10 per student. Students were strongly encouraged to utilize the multimedia content in Moodle, but they made individual decisions on whether or not to benefit from the availability of the content. Some students elected not to benefit at all from the multimedia content, and this subset of the students was a mixture of individuals who achieved quite high marks on quizzes and exams, as well as individuals who achieved rather low marks. On the other hand, other students exceeded the average for accessing the content by a considerable margin. Some students accessed the multimedia content 20 or more times during the term.

ELEC 274, on the other hand, yielded somewhat different results. The students enrolled in this course for the period January-April 2012 were largely the same students from the digital systems course in the immediately preceding term. ELEC 274 has a dedicated laboratory component, and the new exercises produced during this project directed students to view the multimedia content as preparation for their laboratory sessions. These factors led to over 200 accesses of the multimedia content in Moodle by the students during the first week of ELEC 274, just prior to the initial laboratory sessions for the course at the beginning of the second week. For the entire term, Moodle recorded well over 1500 total accesses by students. The enrolment in ELEC 274 was 102 students, which was somewhat less than the preceding course. Hence, the average number of accesses per student was just over 15 . Some individual students made 30 or more accesses.

\section{STUDENT FEEDBACK}

To explore student perspectives on their overall learning experiences in ELEC 271 and ELEC 274, in-class surveys were administered near the end of the term in each course. The responses to the two questions relevant to multimedia content are presented here. Students were asked for a self-assessment on how much of the multimedia content they viewed, and they were asked for their perception of the learning benefit for that content.

For ELEC 271, the survey response rate was $62 \%$ out of 123 students. The graphs in Fig. 5 show the number of 
responses for each choice of the two questions. A small minority of respondents indicated that they did not view the multimedia content and/or did not find it beneficial. The majority of respondents, however, provided a more positive assessment of content utilization and benefit.

How much of multimedia content viewed:

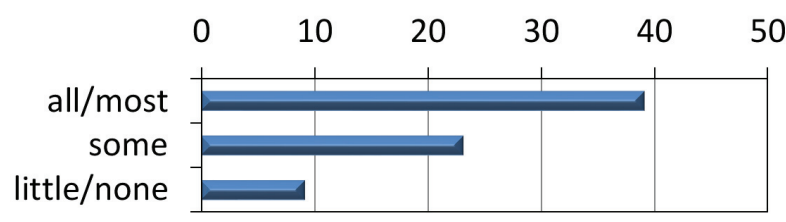

Usefulness for learning enhancement:

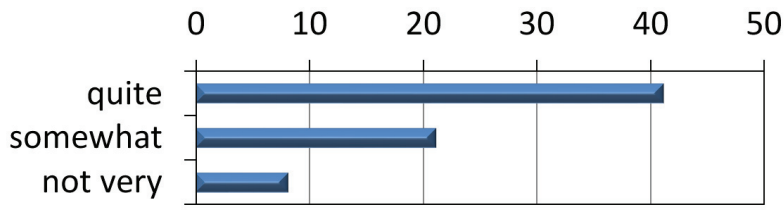

Fig. 5. ELEC 271 student survey results on multimedia

For ELEC 274, the survey response rate was $48 \%$ out of 102 students, and the graphs in Fig. 6 summarize the results for the relevant questions. On a proportional basis, the results for ELEC 274 are more positive than those for the preceding course. A larger majority of students reported making good use of the multimedia content with greater learning benefit. The differences in the subjective responses between the two courses align with the differences in the objective results in Section 6 obtained from monitoring actual usage of the multimedia content.

How much of multimedia content viewed:

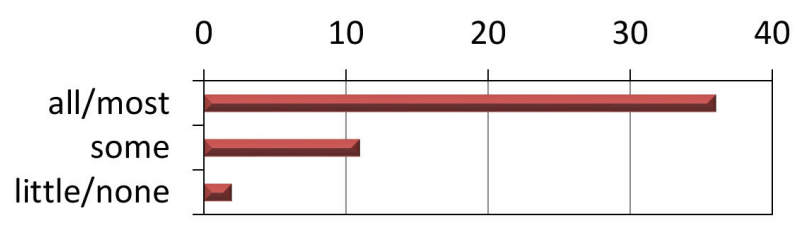

Usefulness for learning enhancement:

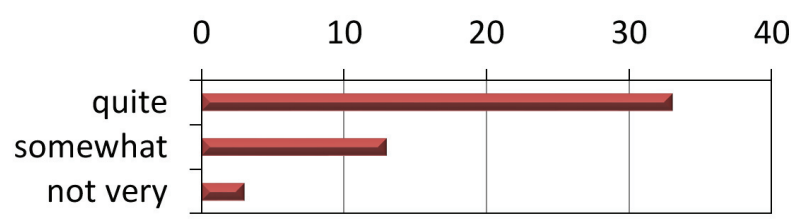

Fig. 6. ELEC 274 student survey results on multimedia

\section{CONCLUSIONS}

This project for learning enhancements in courses on digital systems and computer architecture involved the creation of 24 multimedia presentations and 6 laboratory exercises to improve learning, to link material in different courses, and to provide guidance on practical application of concepts. Tracking for usage of multimedia content by students revealed almost 10 and over 15 average accesses per student in the two relevant courses. Based on feedback from in-class surveys at the end of each course, student perceptions on the usefulness of the multimedia content are positive. With some evidence of students not using the content, there is an opportunity to encourage those students who would face learning challenges in these courses to make the modest commitment of time to use the multimedia content for their benefit. Additional multimedia content can also be produced in future, building on the experience and tools from this project.

\section{Acknowledgements}

A Teaching and Learning Enhancement Grant in 2011 from the Queen's University Centre for Teaching and Learning supported the contributions of Valerie Sugarman, who was at that time a student in the Department of Electrical and Computer Engineering.

\section{References}

[1] Stephen Brown and Zvonko Vranesic, Fundamentals of Digital Logic with VHDL Design. New York, NY: McGrawHill, 2009 (3rd ed.), 939 pp. \{ISBN: 978-0-07-722143-0\}

[2] Brian P. Butz, Michael Duarte, and Susan M. Miller, "An Intelligent Tutoring System for Circuit Analysis," IEEE Trans. on Education., vol. 49, no. 2, pp. 216-223, May 2006.

[3] Carl Hamacher, Zvonko Vranesic, Safwat Zaky, and Naraig Manjikian, Computer Organization and Embedded Systems. New York, NY: McGraw-Hill, 2012 (6th ed.), 736 pp. \{ISBN: 978-0-07-338065-0\}

[4] Corrado Poli, Donald Fisher, Alexander Pollatsek, and Beverly Park Woolf, "Design for Stamping: Identifying Pedagogically Effective Components in Multimedia Tutors and the Classroom," J. of Engineering Education, vol. 92, no. 3, pp. 227-237, July 2003.

[5] Sergio L. Toral Marín, Federico J. Barrero García, Rocío Martínez Torres, Sergio Gallardo Vázquez, and Antonio Javier Lillo Moreno, "Implementation of a Web-Based Educational Tool for Digital Signal Processing Teaching Using the Technological Acceptance Model," IEEE Trans. Edu., vol. 48, no. 4, pp. 632-641, November 2005.

[6] http://www.altera.com/devices/processor/nios2/ni2index.html. 